\title{
The trans-effect of heteroatom in terms of through-space and through-bond interactions. Comparison to the inductive effect
}

\author{
V. Gineityte* \\ Institute of Theoretical Physics and Astronomy, Gostauto 12, 2600 Vilnius, Lithuania
}

Received 17 March 2000; accepted 5 April 2000

\begin{abstract}
The paper is devoted to the investigation of the trans-effect of a heteroatom $\mathrm{Z}$ in substituted alkanes and alkenes revealing itself as non-equivalence of the cis-and trans-arranged $\mathrm{C}_{\beta}-\mathrm{C}_{\gamma}\left(\right.$ or $\left.\mathrm{C}_{\beta}-\mathrm{H}\right)$ bonds with respect to the $\mathrm{Z}-\mathrm{C}_{\alpha}$ bond. To this end, we invoke the power series for the bond order matrix obtained previously in terms of submatrices describing the direct (throughspace) and indirect (through-bond) interorbital interactions (V. Gineityte, J. Mol. Struct. (Theochem) 343 (1995) 183; 364 (1996) $85 ; 434$ (1998) 43). The expressions for the third order corrections to occupation numbers are additionally derived that are absent in the previous contributions but necessary for the investigation of the trans-effect. Two additive components are revealed within the trans-effect, namely the inter-bond charge transfer and the induced dipole moment of the $\mathrm{C}_{\beta}-\mathrm{C}_{\gamma}$ (or $\mathrm{C}_{\beta}-\mathrm{H}$ ) bond under the influence of the $\mathrm{Z}-\mathrm{C}_{\alpha}$ bond. These components and thereby the whole effect are expressed and interpreted in terms of through-space and through-bond interactions of bond orbitals. Comparison of the trans-effect of heteroatom to the inductive effect also is carried out. (C) 2000 Elsevier Science B.V. All rights reserved.
\end{abstract}

Keywords: Trans-effect; Through-space and through-bond interactions; Inductive effect; Heteroatom; Substituted alkanes and alkenes

\section{Introduction}

Analysis of the IR and NMR spectra of numerous substituted alkanes and alkenes [1-4] indicates a nonequivalence of the cis- and trans- $\mathrm{C}_{\beta}-\mathrm{C}_{\gamma}\left(\right.$ or $\left.\mathrm{C}_{\beta}-\mathrm{H}\right)$ bonds with respect to the $\mathrm{Z}-\mathrm{C}_{\alpha}$ bond ( $\mathrm{Z}$ stands here for a heteroatom (substituent)). Moreover, a predominant influence of the heteroatom upon the transarranged bonds, usually referred to as the trans-effect, has been established.

The dependence of the strength of the heteroatom influence on the spatial arrangement of the involved bonds has been supported also by different populations of orbitals of the $\mathrm{C}_{\gamma}(\mathrm{H})$ atoms in the cis- and

\footnotetext{
* Tel.: +370-620-953; fax: +370-222-5361.

E-mail address: gineityte@itpa.lt (V. Gineityte).
}

trans-arranged $\mathrm{C}_{\beta}-\mathrm{C}_{\gamma}$ (or $\mathrm{C}_{\beta}-\mathrm{H}$ ) bonds following from quantum-chemical calculations of electronic structures of substituted hydrocarbons [5-7].

Non-equivalence of the cis-and trans $-\mathrm{C}_{\beta}-\mathrm{C}_{\gamma}$ (or $\mathrm{C}_{\beta}-\mathrm{H}$ ) bonds in substituted hydrocarbons is expected to yield some significant chemical consequences. In particular, the trans-effect of heteroatom is assumed to determine the stereochemistry of the $\beta$-elimination reaction [8]. The predominant trans $\beta$-elimination reported for numerous compounds [9-12] serves to support the above expectation.

Theoretical studies of the nature of trans-effect have been carried out in Refs. [5,13-15]. To this end, the extents of the intramolecular charge transfer between the $\mathrm{Z}-\mathrm{C}_{\alpha}$ and $\mathrm{C}_{\beta}-\mathrm{C}_{\gamma}$ (or $\mathrm{C}_{\beta}-\mathrm{H}$ ) bonds have been compared for the cases of their cis- and transarrangements. 
Special approaches orientated mostly towards investigation of the very trans-effect have been developed and applied in Refs. [5,13,14]. In the starting contribution [5], the so-called generalized principle of the maximum overlap [16] has been adapted for the investigation of the trans-effect. Alternatively, certain topological matrices (adjacency matrices of certain graphs) reflecting the overlap topology of bond orbitals (BOs) of $\sigma$-electron systems (subsystems) of substituted hydrocarbons have been proposed and applied in Refs. [13,14].

The results of these investigations showed that different signs of overlap integrals and thereby of resonance parameters between BOs of the cis- and trans-arranged pairs of vicinal bonds play the principal role in the formation of the trans-effect. It should be mentioned in this context that the absolute values of these overlap integrals were established to fall close together [5,13-15]. Furthermore, similarity of manifestation of the trans-effect in substituted alkanes and alkenes has been supported [13,14], and $\sigma$-electron subsystems were established to be entirely responsible for the effect in the latter case.

In Ref. [15], the origin of the trans-effect of heteroatom has been studied from a more general point of view. To this end, the perturbation theory (PT) for the one-electron density matrix (DM) [17] has been applied. Elements of the DM have been expressed in this PT directly in terms of elements of the initial one-electron Hamiltonian matrix. General nature of this approach may be illustrated by its successful application also to the heteroatom influence upon the nearest $\left(\mathrm{C}_{\alpha}-\mathrm{C}_{\beta}\right)$ bonds in saturated molecules [18-20].

The results of Ref. [15] supported the principal role of signs of overlap integrals between BOs of vicinal bonds in the formation of the trans-effect. Moreover, application of the PT of Ref. [17] allowed us to reveal some common features of the inductive and of the trans-effect of heteroatom. In particular, certain fragment of molecule and not the whole system proved to be responsible for the emergence of the trans-effect as it was the case with the inductive effect [18-20]. On the whole, consideration of the trans-effect in the general context of the heteroatom influence in substituted hydrocarbons proved to be a fruitful approach.

Further development of the PT for the one-electron DM [21] allowed the bond order matrix $P$ to be expressed in terms of two principal submatrices $G_{(1)}$ and $G_{(2)}$, describing the direct (through-space) and indirect (through-bond) interactions of BOs, respectively. This new form of the power series for the matrix $P$ offered additional possibilities for interpretation of particular elements of the DM.

Application of the PT developed in Refs. [21,22] for investigation of the inductive effect [23,24] showed that the influence of heteroatom upon its nearest environment may be described by second order terms of the power series for occupation numbers and consists of two additive components, viz. of the interbond charge transfer and of the secondary polarization of the $\mathrm{C}-\mathrm{C}(\mathrm{C}-\mathrm{H})$ bonds. Moreover, each of these components has been related to definite type of direct and/or indirect interactions of BOs. In particular, the induced dipole moments of $\mathrm{C}-\mathrm{C}(\mathrm{C}-\mathrm{H})$ bonds have been expressed as differences in the indirect interactions between the two BOs of the given bond before and after substitution [24].

In this paper we are about to apply the approach of Refs. [21-24] for investigation of the trans-effect of heteroatom. The main problems arising in this field are as follows: What are the additive components of the trans-effect? Do they resemble those of the inductive effect? Is it possible to interpret these components and thereby the trans-effect itself in terms of direct (through-space) and indirect (through-bond) interactions of BOs? What is the role of signs of overlap integrals between BOs of $\mathrm{Z}-\mathrm{C}_{\alpha}$ and $\mathrm{C}_{\beta}-\mathrm{C}_{\gamma}\left(\mathrm{C}_{\beta}-\mathrm{H}\right)$ bonds in such a new interpretation of the trans-effect? It is precisely the solution of these problems that this paper is aimed at.

In Section 2 of the paper we intend to show that the second order terms of the power series for the oneelectron DM [21,22] are not sufficient when studying the trans-effect. In this connection, the expressions for the third order contributions to occupation numbers of basis orbitals will be additionally derived. Particular additive components of the trans-effect will be analyzed in Sections 3 and 4.

\section{The expressions for populations of basis orbitals of the $\mathrm{C}-\mathrm{C}(\mathrm{C}-\mathrm{H})$ bonds in heteroatom-containing molecules}

The power series for the one-electron DM (bond 


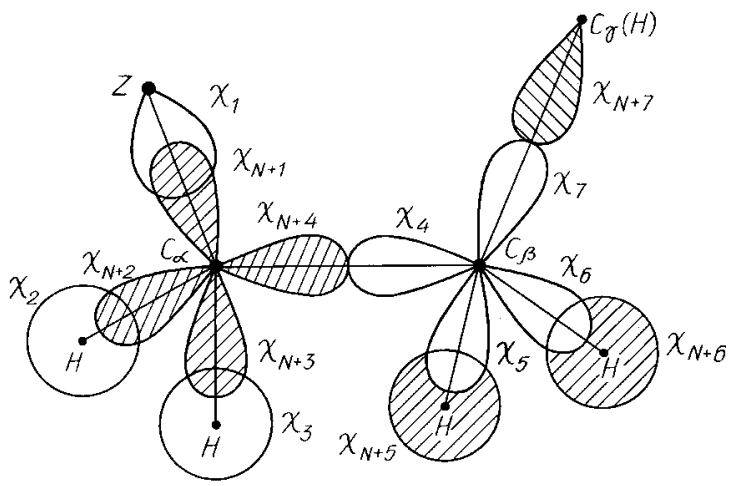

Fig. 1. The numbering of basis orbitals (i.e. of $\mathrm{sp}^{3}$-hybrid AOs and $1 \mathrm{~s}_{\mathrm{H}} \mathrm{AOs}$ ) of a substituted alkane $\mathrm{ZC}_{\alpha} \mathrm{H}_{2} \mathrm{C}_{\beta} \mathrm{H}_{2} \mathrm{C}_{\gamma} \cdots$, where $\mathrm{Z}$ stands for a heteroatom. Dashed orbitals are those described by negative coefficients within the antibonding BOs, and $\mathrm{N}$ coincides with the total number of bonds in molecule.

order matrix) developed in Ref. [21] refers to the basis of BOs, a precise definition which was not required when deriving the general expressions for separate terms of this series. To obtain the occupation numbers of $\mathrm{sp}^{3}$-hybrid AOs of carbon atoms and $1 \mathrm{~s}_{\mathrm{H}}$ AOs of hydrogen atoms in alkanes [22] and their derivatives [24], the above-mentioned BOs have been defined as bonding and antibonding linear combinations of pairs of $\mathrm{sp}^{3}$-hybrid AOs and $1 \mathrm{~s}_{\mathrm{H}}$ AOs corresponding to separate bonds. It is evident that the results of Refs. [21-24] are straightforwardly applicable also to $\sigma-$ electron sub-systems of both alkenes and their derivatives. However, $\mathrm{sp}^{2}$-hybrid AOs should be substituted for $\mathrm{sp}^{3}$-hybrid AOs in this case. Thus, let the abbreviation HAOs stand for either $\mathrm{sp}^{2}$ - or $\mathrm{sp}^{3}$-hybrid AOs.

The expressions for occupation numbers have been derived in Refs. [21-24] to within the second order terms inclusive. The third order terms of the power series for the matrix $P$ represented in the basis of BOs also may be obtained as described in Ref. [21]. To this end, a third order analogue of matrices $G_{(1)}$ and $G_{(2)}$, viz. the matrix $G_{(3)}$ describing the indirect interactions of BOs by means of two mediators, should be introduced. Transformation of terms of this power series into the basis of HAOs also meets no difficulties [22,24].

Let the HAOs and $1 \mathrm{~s}_{\mathrm{H}}$ AOs of a substituted alkane (alkene) containing $N$ bonds to be enumerated as shown in Fig. 1 (For the sake of simplicity, the heteroatom $\mathrm{Z}$ is represented by a single $\mathrm{HAO} \chi_{1}$ direc- ted along the $\mathrm{Z}-\mathrm{C}_{\alpha}$ bond). Then the occupation numbers of HAOs $\chi_{i}$ and $\chi_{N+i}(i \neq 1)$ referring to $\mathrm{C}-\mathrm{C}(\mathrm{C}-\mathrm{H})$ bonds may be expressed as follows:

$X_{i}\left(X_{N+i}\right)=1+\frac{1}{2} \Delta X_{(2) I} \pm p_{(2) I}+\frac{1}{2} \Delta X_{(3) I} \pm p_{(3) I}$

where the subscripts (2) and (3) denote the second and the third order increments, respectively, and $I$ stands for the bond the orbitals $\chi_{i}$ and $\chi_{N+i}$ belong to. The upper signs of the right-hand side of Eq. (1) refer to the HAO $\chi_{i}$, whereas the lower ones correspond to $\chi_{N+i}$.

Let us consider the terms of Eq. (1) separately. The only zero order term equal to 1 represents the initial occupation number of an isolated orbital. The second order term (1/2) $\Delta X_{(2) I}$ describes a half of the total population of the Ith bond lost (acquired) by this bond owing to the interbond charge transfer [24]. This term may be represented as a sum of contributions $\Delta X_{(2) I(J)}$, each of them associated with certain $(J$ th) bond that plays the role of an acceptor (donor) of the lost (acquired) population. The total population alteration $\Delta X_{(2) I}$ takes the form

$\Delta X_{(2) I}=\sum_{J} \Delta X_{(2) I(J)}=2 \sum_{j \ni J}\left\{\left(G_{(1) j i}\right)^{2}-\left(G_{(1) i j}\right)^{2}\right\}$

where the subscript $j$ embraces the two BOs of the $J$ th bond, viz. the bonding $\mathrm{BO} \varphi_{(+) j}$ and the antibonding $\operatorname{BO} \varphi_{(-) j}$. The notation $G_{(1) i j}$ stands for an element of the matrix $G_{(1)}$ [21] describing the direct (throughspace) interaction between the bonding $\mathrm{BO}$ (BBO) $\varphi_{(+) i}$ of the Ith bond and the antibonding $\mathrm{BO}(\mathrm{ABO})$ $\varphi_{(-) j}$ of the $J$ th bond.

As in Ref. [24], let us introduce the following notations for resonance parameters between various types of BOs, the latter being indicated within the bra-and ket-vectors, viz.

$S_{i j}=\left\langle\varphi_{(+) i}|\hat{H}| \varphi_{(+) j}\right\rangle, \quad R_{i j}=\left\langle\varphi_{(+) i}|\hat{H}| \varphi_{(-) j}\right\rangle$,

$Q_{i j}=\left\langle\varphi_{(-) i}|\hat{H}| \varphi_{(-) j}\right\rangle$

and note that $S_{i j}=S_{j i}, Q_{i j}=Q_{j i}$ but $R_{i j} \neq R_{j i}$. Then the element $G_{(1) i j}$ takes the form

$G_{(1) i j}=-\frac{R_{i j}}{E_{(+) i}-E_{(-) j}}$

where the denominator of Eq. (4) contains the difference in one-electron energies of BOs $\varphi_{(+) i}$ and $\varphi_{(-) j}$.

Let us turn again to Eq. (2). It is seen that the 
increment of the $J$ th bond to the total population lost (acquired) by the Ith bond depends on the difference between squares of the direct (through-space) interactions of the $\mathrm{BBO} \varphi_{(+) i}$ and $\mathrm{ABO} \varphi_{(-) j}$, on the one hand, and of the $\operatorname{BBO} \varphi_{(+) j}$ and $\operatorname{ABO} \varphi_{(-) i}$, on the other hand. This implies that the contribution of the $J$ th bond to the population of the Ith bond actually depends only on the mutual arrangement of two bonds and does not depend on the remaining fragment of the molecule.

The next term of Eq. (1) $\left(p_{(2) I}\right)$ describes the second order induced dipole moment of the Ith bond under the influence of other bonds. This dipole moment may be expressed as follows:

$p_{(2) I}=-2 G_{(2) i i}$

where $G_{(2) i i}$ is the diagonal element of the second order matrix $G_{(2)}$ [21] representing the indirect (through-bond) interactions of BOs. For further convenience, any element $G_{(2) i j}$ is given below in Eq. (6)

$$
\begin{aligned}
G_{(2) i j}= & \frac{1}{E_{(+) i}-E_{(-) j}} \\
& \times\left(\sum_{l}^{\mathrm{BBOs}} \frac{S_{i l} R_{l j}}{E_{(+) l}-E_{(-) j}}-\sum_{k}^{\mathrm{ABOs}} \frac{R_{i k} Q_{k j}}{E_{(+) i}-E_{(-) k}}\right)
\end{aligned}
$$

and the diagonal element $G_{(2) i i}$ follows from Eq. (6) if $i=j$. The notations of Eq. (6) coincide with those of Eq. (4). From Eqs. (5) and (6) it may be easily seen that the induced dipole moment $p_{(2) I}$ is determined by the indirect interaction of BOs $\varphi_{(+) i}$ and $\varphi_{(-) i}$ of the $I$ th bond by means of BBOs and/or ABOs of other bonds.

Constitution of Eq. (6) shows that the dipole moment $p_{(2) I}$ also may be presented as a sum of particular increments $p_{(2)(J)}$, where

$$
\begin{aligned}
p_{(2) I(J)}= & -2 \frac{1}{E_{(+) i}-E_{(-) i}} \\
& \times\left(\frac{S_{i j} R_{j i}}{E_{(+) j}-E_{(-) i}}-\frac{R_{i j} Q_{j i}}{E_{(+) i}-E_{(-) j}}\right)
\end{aligned}
$$

It is seen that this increment also depends on the mutual arrangement of the $I$ th and $J$ th bonds only.

It should be noted here that the corrections $\Delta X_{(2) I}$ and $p_{(2) I}$ defined by Eqs. (2) and (5) have been success- fully applied for investigation of the inductive effect [23,24]: These corrections described the additive components of the heteroatom influence upon the nearest $\left(\mathrm{C}_{\alpha}-\mathrm{C}_{\beta}\right)$ bonds.

Before turning to the third order increments of Eq. (1), let us compare the corrections $\Delta X_{(2) I}$ and $p_{(2) I}$ for cis- and trans-arranged $\mathrm{C}_{\beta}-\mathrm{C}_{\gamma}\left(\mathrm{C}_{\beta}-\mathrm{H}\right)$ bonds.

In accordance with Fig. 1 , the bond $I=7$ corresponds to the $\mathrm{C}_{\beta}-\mathrm{C}_{\gamma}\left(\mathrm{C}_{\beta}-\mathrm{H}\right)$ bond. If we are interested only in terms arising as a result of substitution, differences between occupation numbers of respective basis orbitals of the substituted molecule and those of the parent hydrocarbon should be considered. It is evident that only terms corresponding to $J=1$ remain in these differences.

As a result, the influence of the heteroatom $\mathrm{Z}$ upon the population lost (acquired) by the $\mathrm{C}_{\beta}-\mathrm{C}_{\gamma}\left(\mathrm{C}_{\beta}-\mathrm{H}\right)$ bond equals to

$$
\begin{aligned}
\delta\left(\Delta X_{(2) 7}\right)= & \delta\left(\Delta X_{(2) 7(1)}\right)=\Delta X_{(2) 7(1)}-\Delta X_{(2) 7(1)}^{\mathrm{o}} \\
= & 2\left\{\left(G_{(1) 17}\right)^{2}-\left(G_{(1) 71}\right)^{2}\right\}-2\left\{\left(G_{(1) 17}^{\mathrm{o}}\right)^{2}\right. \\
& \left.-\left(G_{(1) 17}^{\mathrm{o}}\right)^{2}\right\}
\end{aligned}
$$

where the superscript o here and below refers to the parent hydrocarbon.

It is seen that the population alteration $\delta\left(\Delta X_{(2) 7}\right)$ depends only on absolute values of the throughspace interactions between BOs of the $\mathrm{Z}-\mathrm{C}_{\alpha}$ and $\mathrm{C}_{\beta}-\mathrm{C}_{\gamma}\left(\mathrm{C}_{\beta}-\mathrm{H}\right)$ bonds, but it is independent of signs of these interactions. Inasmuch as absolute values of resonance parameters $R_{71}$ and of $R_{17}$ were established to coincide within a few percent for cis- and transarranged $\mathrm{Z}-\mathrm{C}_{\alpha}$ and $\mathrm{C}_{\beta}-\mathrm{C}_{\gamma}\left(\mathrm{C}_{\beta}-\mathrm{H}\right)$ bonds [5,13-15], the absolute values of the respective through-space interactions of BOs fall close together. As a result, a negligible difference between the population alterations $\delta\left(\Delta X_{(2) 7}\right)^{c i s}$ and $\delta\left(\Delta X_{(2) 7}\right)^{\text {trans }}$ follows.

To compare the second order dipole moments of cis- and trans $-\mathrm{C}_{\beta}-\mathrm{C}_{\gamma}\left(\mathrm{C}_{\beta}-\mathrm{H}\right)$ bonds arising under the influence of heteroatom, the difference $\delta p_{(2) 7}$ should be studied.

Let the energy reference point $(\alpha)$ coincide with the average value of one-electron energies of HAOs and $1 \mathrm{~s}_{\mathrm{H}}$ AOs, whereas the energy unit $(\beta)$ will be equal to the mean value of resonance parameters for pairs of HAOs and $1 \mathrm{~s}_{\mathrm{H}}$ AOs of the same bond. Furthermore, let us accept the equalities $\alpha=0$ and $\beta=1$ for 

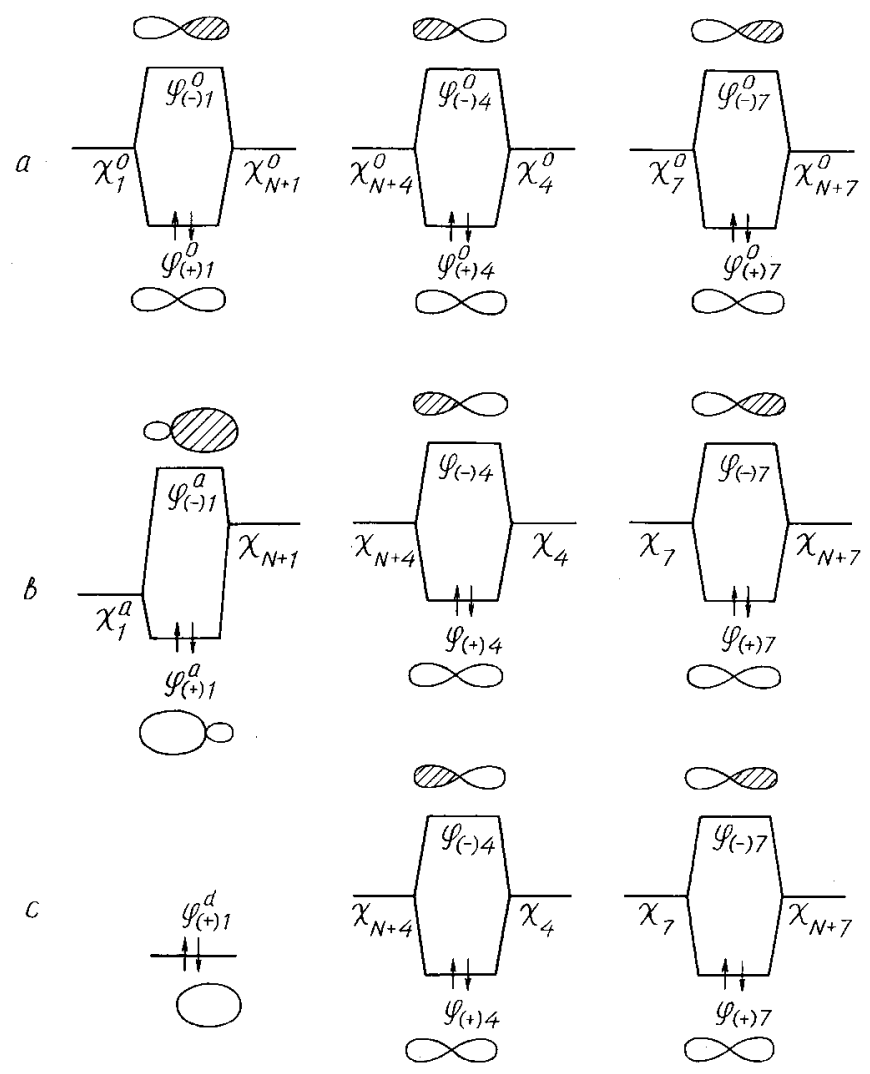

Fig. 2. Diagram reflecting the one-electron energies of BBOs $\varphi_{(+) i}$ and of ABOs $\varphi_{(-) i}, i=1,4,7$ of the Z- $C_{\alpha}, C_{\alpha}-C_{\beta}$ and $C_{\beta}-C_{\gamma}\left(C_{\beta}-H\right)$ bonds, and the shapes of these orbitals. The first picture (a) corresponds to the parent hydrocarbon $(\mathrm{Z}=\mathrm{H})$, whereas substituted alkanes (alkenes) containing an electron-accepting and electron-donating substituents are represented by pictures (b) and (c), respectively.

convenience. As a result, the energy differences within denominators of Eqs. (4) and (6) are positive and the same refers to resonance parameters within numerators provided that the respective overlap integrals are positive. Using Eq. (7) we then obtain

$$
\begin{aligned}
\delta p_{(2) 7}= & p_{(2) 7}-p_{(2) 7}^{\mathrm{o}}=p_{(2) 7(1)}-p_{(2) 7(1)}^{\mathrm{o}} \\
= & \frac{1}{2} S_{71}^{\mathrm{o}} R_{17}^{\mathrm{o}}-\frac{1}{2} R_{71}^{\mathrm{o}} Q_{17}^{\mathrm{o}}-\frac{S_{71} R_{17}}{E_{(+) 1}-E_{(-) 7}} \\
& +\frac{R_{71} Q_{17}}{E_{(+) 7}-E_{(-) 1}}
\end{aligned}
$$

where the differences $E_{(+) 7}-E_{(-) 7}$ and $E_{(+) 7}^{\mathrm{o}}-E_{(-) 7}^{\mathrm{o}}$ are taken equal to 2 [14].

From Eq. (9) it follows that resonance parameters between BOs of $\mathrm{Z}-\mathrm{C}_{\alpha}$ and $\mathrm{C}_{\beta}-\mathrm{C}_{\gamma}\left(\mathrm{C}_{\beta}-\mathrm{H}\right)$ bonds determine the difference $\delta p_{(2) 7}$. As it was mentioned already, these parameters are of different signs for cisand trans-arranged vicinal bonds [13-16].

Let the BBOs and $\mathrm{ABOs}$ to be defined as follows [24]:

$$
\varphi_{(+) i}=a \chi_{i}+b \chi_{N+i}, \quad \varphi_{(-) i}=b \chi_{i}-a \chi_{N+i}
$$

where $a$ and $b$ are numerical coefficients coinciding with $(1 / \sqrt{2})$ for $\mathrm{C}-\mathrm{C}(\mathrm{C}-\mathrm{H})$ bonds.

Then the increments $\left\langle\chi_{7}|\hat{H}| \chi_{N+1}\right\rangle$ make the principal contributions to resonance parameters between BOs of vicinal bonds. As a result, the following inequalities:

$$
\begin{aligned}
& S_{71}^{\text {cis }}>0, \quad S_{71}^{\text {trans }}<0, \quad R_{17}^{\text {cis }}>0, \quad R_{17}^{\text {trans }}<0, \\
& R_{71}^{\text {cis }}<0, \quad R_{71}^{\text {trans }}>0, \quad Q_{17}^{\text {cis }}<0, \quad Q_{17}^{\text {trans }}>0
\end{aligned}
$$

may be easily established on the basis of Fig. 2, and 
these refer to the resonance parameters of the parent hydrocarbon as well.

It is seen that all resonance parameters contained within Eq. (9) change their signs if we turn from the cis-arranged $\mathrm{C}_{\beta}-\mathrm{C}_{\gamma}\left(\mathrm{C}_{\beta}-\mathrm{H}\right)$ bond to the transarranged one. Hence, the difference $\delta p_{(2) 7}$ proves to be independent of the spatial arrangement of the $\mathrm{Z}-\mathrm{C}_{\alpha}$ and $\mathrm{C}_{\beta}-\mathrm{C}_{\gamma}\left(\mathrm{C}_{\beta}-\mathrm{H}\right)$ bonds, provided that coinciding absolute values of respective resonance parameters are assumed [5,13-15].

Therefore, to reveal the differences between populations of the cis- and trans-arranged $\mathrm{C}_{\beta}-\mathrm{C}_{\gamma}\left(\mathrm{C}_{\beta}-\mathrm{H}\right)$ bonds, third order terms of Eq. (1) should be considered. Thus, let us revert to Eq. (1) again.

The increment $\Delta X_{(3) I}$ describes the third order contribution to the population lost (acquired) by the Ith bond owing to the interbond charge transfer. As with the respective second order increment $\Delta X_{(2) I}$, this term is additive with respect to contributions of separate bonds and takes the form

$$
\Delta X_{(3) I}=\sum_{J} \Delta X_{(3) I(J)}=4 \sum_{j \ni J}\left(G_{(1) j i} G_{(2) j i}-G_{(1) i j} G_{(2) i j}\right)
$$

where $G_{(2) i j}$ is defined by Eq. (6). It is seen that products of direct and of indirect interactions of BOs $\varphi_{(+) i}$ and $\varphi_{(-) j}$, as well as of $\varphi_{(-) i}$ and $\varphi_{(+) j}$, are present within Eq. (12) in contrast to Eq. (2). Another essential difference between $\Delta X_{(2) I}$ and $\Delta X_{(3) I}$ consists in the fact that the contribution of the $J$ th bond to the third order term $\Delta X_{(3) I}$ depends not only on the spatial arrangement of the $I$ th and $J$ th bonds, but also on that of other bonds the orbitals of which play the role of mediators in the indirect interactions represented by the elements $G_{(2) i j}$ and $G_{(2) j i}$. Hence, the third order interbond charge transfer is essentially non-local in nature.

The last term of Eq. (1), viz. $p_{(3) I}$, yields a dipolelike contribution to populations of orbitals $\chi_{i}$ and $\chi_{N+i}$. Thus, it may be interpreted as the third order induced dipole moment of the Ith bond. This dipole moment proves to be proportional to the indirect interaction between the $\mathrm{BBO} \varphi_{(+) i}$ and $\operatorname{ABO} \varphi_{(-) i}$ of the Ith bond by means of two mediators, viz.

$p_{(3) I}=-2 G_{(3) i i}$ where

$$
\begin{aligned}
G_{(3) i i}= & \frac{-1}{E_{(+) i}-E_{(-) i}} \\
& \times\left\{\sum_{j}^{\mathrm{BBOs}} \sum_{m}^{\mathrm{BBOs}} \frac{S_{i j} S_{j m} R_{m i}}{\left(E_{(+) j}-E_{(-) i}\right)\left(E_{(+) m}-E_{(-) i}\right)}\right. \\
& -\sum_{j}^{\mathrm{BBOS}} \sum_{r}^{\mathrm{ABOs}}\left[\frac{S_{i j} R_{j r} Q_{r i}}{\left(E_{(+) j}-E_{(-))}\right)\left(E_{(+) j}-E_{(-) r}\right)}\right. \\
& +\frac{S_{i j} R_{j r} Q_{r i}}{\left(E_{(+) i}-E_{(-) r}\right)\left(E_{(+) j}-E_{(-) r}\right)} \\
& +\frac{R_{i r} R_{r j}^{+} R_{j i}}{\left(E_{(+) j}-E_{(-) r}\right)\left(E_{(+) j}-E_{(-) i}\right)} \\
& \left.+\frac{R_{i r} R_{r j}^{+} R_{j i}}{\left(E_{(+) i}-E_{(-) r}\right)\left(E_{(+) j}-E_{(-) r}\right)}\right] \\
& \left.+\sum_{p}^{\mathrm{ABOs}} \sum_{r}^{\mathrm{ABOs}} \frac{R_{i r} Q_{r p} Q_{p i}}{\left(E_{(+) i}-E_{(-) p}\right)\left(E_{(+) i}-E_{(-) r}\right)}\right\}
\end{aligned}
$$

and $R_{r j}^{+}=R_{j r}$. Non-local nature of particular increments to the third order dipole moment $p_{(3) I}$ is evident.

The third order terms $\Delta X_{(3) I}$ and $p_{(3) I}$ for cis- and trans-arranged $\mathrm{C}_{\beta}-\mathrm{C}_{\gamma}\left(\mathrm{C}_{\beta}-\mathrm{H}\right)$ bonds with respect to the $\mathrm{Z}-\mathrm{C}_{\alpha}$ bond will be studied separately in Sections 3 and 4 , respectively.

\section{The third order interbond charge transfer between the $\mathrm{Z}-\mathrm{C}_{\alpha}$ and $\mathrm{C}_{\boldsymbol{\beta}}-\mathrm{C}_{\gamma}\left(\mathrm{C}_{\boldsymbol{\beta}}-\mathrm{H}\right)$ bonds}

Let us start with an electron-accepting substituent $Z_{a}$ described by a higher electronegativity as compared to those of hydrogen and carbon atoms. Relative positions of one-electron energies of BOs and their shapes [24] are displayed in Fig. $2 b$.

To compare the third order increments to the population of the bond $I=7$ lost (acquired) from other bonds, the difference

$$
\delta\left(\Delta X_{(3) 7}^{(\mathrm{a})}\right)=\Delta X_{(3) 7}^{(\mathrm{a})}-\Delta X_{(3) 7}^{\mathrm{o}}
$$

should be considered, where the superscript (a) here 
and below refers to the electron-accepting substituent $\mathrm{Z}_{\mathrm{a}}$.

It may be easily seen from Eqs. (4), (6) and (12) that two types of terms remain within the difference of Eq. (15), namely terms corresponding to $J=1$ (as it was the case with the second order charge transfer) and terms wherein the orbitals $\varphi_{(+) 1}$ and $\varphi_{(-) 1}$ of the 1 st bond play the role of mediators of the indirect interactions $G_{(2) i j}$ and $G_{(2) j i}$ between orbitals of the $I$ th and $J$ th bond $(J \neq 1$ in the latter case).

Let us start with terms corresponding to $J=1$ and describing the increment of the $\mathrm{Z}_{\mathrm{a}}-\mathrm{C}_{\alpha}\left(\mathrm{H}-\mathrm{C}_{\alpha}\right)$ bond to the total correction $\delta\left(\Delta X_{(3) 7}^{(\mathrm{a})}\right)$. This increment easily follows from Eq. (12), viz.

$$
\begin{gathered}
\Delta X_{(3) 7(1)}^{(\mathrm{a})}-\Delta X_{(3) 7(1)}^{\mathrm{o}}=4\left(G_{(1) 17} G_{(2) 17}-G_{(1) 71} G_{(2) 71}\right) \\
-4\left(G_{(1) 17}^{\mathrm{o}} G_{(2) 17}^{\mathrm{o}}-G_{(1) 71}^{\mathrm{o}} G_{(2) 71}^{\mathrm{o}}\right)
\end{gathered}
$$

where $G_{(1) 17}, G_{(1) 71}, G_{(1) 17}^{\mathrm{o}}$ and $G_{(1) 71}^{\mathrm{o}}$ are defined by Eq. (4).

To evaluate the elements $G_{(2) 17}, G_{(2) 71}, G_{(2) 17}^{\mathrm{o}}$ and $G_{(2) 71}^{\mathrm{o}}$ of the second order matrix $G_{(2)}$, the expression of Eq. (6) should be used. It is seen that BOs $\left(\varphi_{(+) l}\right.$ and $\left.\varphi_{(-) k}\right)$ that overlap significantly both with the BBO $\varphi_{(+) i}$ and with the $\mathrm{ABO} \varphi_{(-) j}$, are the most efficient mediators for the indirect interaction represented by the element $G_{(2) i i}$. On the other hand, orbitals of the $I$ th and $J$ th bonds do not play the role of mediators as diagonal elements $S_{i i}, R_{i i}$ and $Q_{i i}$ take zero values [23]. Hence, BOs $l=\varphi_{(+) 4}$ and $k=\varphi_{(-) 4}$ of the $\mathrm{C}_{\alpha}-\mathrm{C}_{\beta}$ bond may be expected to play the role of the principal mediators in the indirect interactions both between BOs $\varphi_{(+) 7}$ and $\varphi_{(-) 1}$, and between $\varphi_{(+) 1}$ and $\varphi_{(-) 7}$. With this in mind we then obtain

$$
G_{(2) 17} \approx \frac{1}{E_{(+) 1}-E_{(-) 7}}\left(\frac{S_{14} R_{47}}{E_{(+) 4}-E_{(-) 7}}-\frac{R_{14} Q_{47}}{E_{(+) 1}-E_{(-) 4}}\right)
$$

and

$$
G_{(2) 71} \approx \frac{1}{E_{(+) 7}-E_{(-) 1}}\left(\frac{S_{74} R_{41}}{E_{(+) 4}-E_{(-) 1}}-\frac{R_{74} Q_{41}}{E_{(+) 7}-E_{(-) 4}}\right)
$$

From Eqs. (4), (17) and (18) along with Fig. 2, the following inequalities result for interactions contained within Eq. (16)

$$
\begin{aligned}
& \left|G_{(1) 17}\right| \ll\left|G_{(1) 71}\right|, \quad\left|G_{(2) 17}\right|<\left|G_{(2) 71}\right| \\
& G_{(1) 17}^{\mathrm{o}}=G_{(1) 71}^{\mathrm{o}}, \quad G_{(2) 17}^{\mathrm{o}}=G_{(2) 71}^{\mathrm{o}} .
\end{aligned}
$$

Consequently, the difference shown in the left-hand side of Eq. (16) may be approximated as follows:

$\Delta X_{(3) 7(1)}^{(\mathrm{a})}-\Delta X_{(3) 7(1)}^{\mathrm{o}} \approx \Delta X_{(3) 7(1)}^{(\mathrm{a})} \approx-4 G_{(1) 71} G_{(2) 71}$

The first order factors of Eq. (21) referring to the cis- and trans-arranged $\mathrm{C}_{\beta}-\mathrm{C}_{\gamma}\left(\mathrm{C}_{\beta}-\mathrm{H}\right)$ bonds are of positive and negative values, respectively, i.e.

$G_{(1) 71}^{\text {cis }}>0, \quad G_{(1) 71}^{\text {trans }}<0$

as Eqs. (4) and (11) indicate. On the other hand, from Fig. 2 it is seen that

$S_{74}>0, R_{41}<0, R_{74}>0, Q_{14}>0$

irrespective of the spatial arrangement of the $Z_{a}-C_{\alpha}$ and $\mathrm{C}_{\beta}-\mathrm{C}_{\gamma}\left(\mathrm{C}_{\beta}-\mathrm{H}\right)$ bonds.

This implies that

$G_{(2) 71}^{\text {cis }}<0, \quad G_{(2) 71}^{\text {trans }}<0$.

As a result, Eq. (21) yields the following inequalities:

$\left(\Delta X_{(3) 7(1)}^{(\mathrm{a})}-\Delta X_{(3) 7(1)}^{\mathrm{o}}\right)^{c i s}>0$,
$\left(\Delta X_{(3) 7(1)}^{(\mathrm{a})}-\Delta X_{(3) 7(1)}^{\mathrm{o}}\right)^{\text {trans }}<0$.

Let us turn now to the case $J \neq 1$. Small contributions to the total difference $\delta\left(\Delta X_{(3) 7}^{(\mathrm{a})}\right)$ of Eq. (15) will be obtained in this case owing to small elements $G_{(2) 7 j}$ of the matrix $G_{(2)}$ wherein just the BOs $\varphi_{(+) 1}$ and $\varphi_{(-) 1}$ play the role of mediators in the indirect interaction between BOs $\varphi_{(+) 7}$ and $\varphi_{(-) j}(j \neq 1,7)$. The fact that BOs of the first bond are not efficient mediators for the above-mentioned interaction may be easily supported on the basis of Figs. 1 and 2.

Hence, the inequalities shown in Eq. (25) may be expected to be valid also for total differences of Eq. (15), i.e.

$\delta\left(\Delta X_{(3) 7}^{(\mathrm{a})}\right)^{\text {cis }}>0, \quad \delta\left(\Delta X_{(3) 7}^{(\mathrm{a})}\right)^{\text {trans }}<0$.

It may be concluded, therefore, that the $c i s-\mathrm{C}_{\beta}-$ $\mathrm{C}_{\gamma}\left(\mathrm{C}_{\beta}-\mathrm{H}\right)$ bond acquires an additional population owing to the third order interbond charge transfer in 
molecules containing an electron-accepting substituent, and the total population of this bond becomes relatively increased. Accordingly, the trans $-\mathrm{C}_{\beta}-$ $\mathrm{C}_{\gamma}\left(\mathrm{C}_{\beta}-\mathrm{H}\right)$ bond loses its population owing to the third order charge transfer. This result indicates the trans electron-accepting effect to take place owing to the interbond charge transfer in molecules containing an electronegative substituent.

Let us discuss now the case of an electron-donating substituent $Z_{d}$. This type of substituent may be exemplified by groups of atoms containing lone electron pairs, e.g. the $\mathrm{NH}_{2}$-group in the well-studied methylamine molecule $[5,16]$. Let us assume in this connection that our substituent is described by an initially occupied orbital $\varphi_{(+) 1}^{\mathrm{d}}$ situated above the bonding BOs of the $\mathrm{C}-\mathrm{C}(\mathrm{C}-\mathrm{H})$ bonds (Fig. 2c). Then the analogue of Eq. (21) for an electron-donating substituent takes the form

$\Delta X_{(3) 7(1)}^{(\mathrm{d})}-\Delta X_{(3) 7(1)}^{\mathrm{o}} \approx \Delta X_{(3) 7(1)}^{(\mathrm{d})} \approx 4 G_{(1) 17} G_{(2) 17}$.

Taking Eqs. (4) and (11) into consideration we then obtain

$G_{(1) 17}^{\text {cis }}<0, \quad G_{(1) 17}^{\text {trans }}>0$

On the other hand, using Eq. (17) and the inequalities

$S_{14}>0, R_{47}>0, R_{14}<0, Q_{47}>0$

we obtain that

$G_{(2) 17}^{\text {cis }}>0, \quad G_{(2) 17}^{\text {trans }}>0$

On the basis of Eqs. (27), (28) and (30), the final result follows, viz.

$\delta\left(\Delta X_{(3) 7(1)}^{(\mathrm{d})}\right)^{c i s} \approx \delta\left(\Delta X_{(3) 7}^{(\mathrm{d})}\right)^{c i s}<0$,

$\delta\left(\Delta X_{(3) 7(1)}^{(\mathrm{d})}\right)^{\text {trans }} \approx \delta\left(\Delta X_{(3) 7}^{(\mathrm{d})}\right)^{\text {trans }}>0$.

It is seen, therefore, that the population of the trans$\mathrm{C}_{\beta}-\mathrm{C}_{\gamma}\left(\mathrm{C}_{\beta}-\mathrm{H}\right)$ bond becomes additionally increased vs. that of the cis-arranged bond owing to the third order interbond charge transfer in molecules containing an electron-donating substituent. Consequently, the trans-electron-donating effect may be concluded to take place in these molecules.

On the whole, the results of the above study support the emergence of the trans-effect of heteroatom (substituent) within the third order terms of the power series for the bond order matrix $P$ representing the interbond charge transfer. Moreover, the origin of this effect may be easily discovered on the basis of Eqs. (21) and (27).

Indeed, the extent of the third order interbond charge transfer proves to be determined by the product of the direct and of the indirect interaction between orbitals of the $\mathrm{Z}-\mathrm{C}_{\alpha}$ and $\mathrm{C}_{\beta}-\mathrm{C}_{\gamma}\left(\mathrm{C}_{\beta}-\mathrm{H}\right)$ bonds. The first of these factors changes its sign when turning from the cis- to trans-arranged $\mathrm{C}_{\beta}-\mathrm{C}_{\gamma}\left(\mathrm{C}_{\beta}-\mathrm{H}\right)$ bond, whereas the sign of the second factor is independent of the spatial arrangement of the $\mathrm{Z}-\mathrm{C}_{\alpha}$ and $\mathrm{C}_{\beta}-$ $\mathrm{C}_{\gamma}\left(\mathrm{C}_{\beta}-\mathrm{H}\right)$ bonds. As a result, the signs of the third order charge transfer term are opposite for cis- and trans-arranged bonds, and the trans-effect of heteroatom follows.

The above-drawn conclusions may be easily related to those of Refs. [5,13-15], where the trans-effect of heteroatom was assumed to manifest itself only as an interbond charge transfer. In these contributions, differences in the populations of cis- and trans $-\mathrm{C}_{\beta}-$ $\mathrm{C}_{\gamma}\left(\mathrm{C}_{\beta}-\mathrm{H}\right)$ bonds under the influence of an electronaccepting substituent were shown to be determined by signs of products made up of resonance parameters between the bonding $\mathrm{BO}$ of the bond under study $\left(\varphi_{(+) 7}\right)$ and the antibonding $\mathrm{BO}$ of the $\mathrm{Z}_{\mathrm{a}}-\mathrm{C}_{\alpha}$ bond $\left(\varphi_{(-) 1}\right)$, between the former and the bonding BO of the $\mathrm{C}_{\alpha}-\mathrm{C}_{\beta}$ bond $\left(\varphi_{(+) 4}\right)$, and between the antibonding $\mathrm{BO}$ on the $\mathrm{Z}_{\mathrm{a}}-\mathrm{C}_{\alpha}$ bond $\left(\varphi_{(-) 1}\right)$ and the bonding BO of the $\mathrm{C}_{\alpha}-\mathrm{C}_{\beta}$ bond $\left(\varphi_{(+) 4}\right)$. Using the notations of Eq. (3), the above-described product coincides with $S_{74} R_{41} R_{71}$. The same product also follows from Eq. (21) after taking into account the expressions for $G_{(1) 71}$ (Eq. (4)) and for $G_{(2) 71}$ (Eq. (18)) under an additional assumption that the absolute value of the first term of the right-hand side of Eq. (18) exceeds that of the second term considerably. Two points serve to support the latter expectation, viz. the smaller energy difference of denominator within the first term of Eq. (18) as compared to the second one and the inequalities [12]

$\left|S_{74}\right|>\left|R_{74}\right|, \quad\left|R_{41}\right|>\left|Q_{41}\right|$

For an electron-donating substituent, the analogous product obtained in Ref. [15] is $R_{17} R_{14} Q_{47}$ and it also coincides with that following from Eqs. (27) and (31). Moreover, the conclusion of Ref. [15] about the principal role of the three bonds $\left(\mathrm{Z}_{\mathrm{a}}-\mathrm{C}_{\alpha}, \mathrm{C}_{\alpha}-\mathrm{C}_{\beta}\right.$ and 
$\left.\mathrm{C}_{\beta}-\mathrm{C}_{\gamma}\left(\mathrm{C}_{\beta}-\mathrm{H}\right)\right)$ in the formation of the trans-effect also is borne out by the results of our study.

\section{The third order induced dipole moments for cis- and trans- $\mathrm{C}_{\beta}-\mathrm{C}_{\gamma}\left(\mathrm{C}_{\beta}-\mathrm{H}\right)$ bonds}

In this section, let us consider the last term of Eq. (1) describing the third order dipole moment of the Ith bond.

Let us start again with an electron-accepting substituent $\mathrm{Z}_{\mathrm{a}}$ and take $I=7$. As with the charge transfer term $\Delta X_{(3) I}$, let us consider the difference

$\delta p_{3(7)}=p_{(3) 7}^{(\mathrm{a})}-p_{(3) 7}^{\mathrm{o}}$.

Non-zero increments to this difference necessarily contain the orbitals $\varphi_{(+) 1}$ and $\varphi_{(-) 1}$ of the $\mathrm{Z}_{\mathrm{a}}-\mathrm{C}_{\alpha}$ bond as mediators of the indirect interaction between BOs $\varphi_{(+) 7}$ and $\varphi_{(-) 7}$ represented by the element $G_{(3) 77}$. From Fig. 2 it is seen that the orbital $\varphi_{(-) 1}^{\mathrm{a}}$ is a more efficient mediator as compared to the orbital $\varphi_{(+) 1}^{\mathrm{a}}$. Given that $\varphi_{(-) 1}^{\mathrm{a}}$ is the first mediator of the interaction between BOs $\varphi_{(+) 7}$ and $\varphi_{(-) 7}$, the orbitals of the $\mathrm{C}_{\alpha}-$ $\mathrm{C}_{\beta}$ bond, viz. $\varphi_{(+) 4}$ and $\varphi_{(-) 4}$, play the role of the second mediator (Note that just the orbitals $\varphi_{(+) 4}$ and $\varphi_{(-) 4}$ interact (overlap) directly with all the involved orbitals, viz. $\varphi_{(-) 1}^{\mathrm{a}}, \varphi_{(+) 7}$ and $\left.\varphi_{(-) 7}\right)$.

Consequently, the most significant terms of the dipole $p_{(3) 7}^{(\mathrm{a})}$ contributing to the difference $\delta p_{(3) 7}^{(\mathrm{a})}$ of Eq. (33) are

$$
\begin{aligned}
& p_{(3) 7}^{(\mathrm{a})} \sim-\frac{S_{74} R_{41} Q_{17}}{2\left(E_{(+) 4}-E_{(-) 1}\right)} \\
& -\frac{S_{74} R_{41} Q_{17}}{\left(E_{(+) 7}-E_{(-) 1}\right)\left(\left(E_{(+) 4}-E_{(-) 1}\right)\right.}+\frac{R_{74} Q_{41} Q_{17}}{2\left(E_{(+) 7}-E_{(-) 1}\right)} \\
& +\frac{R_{71} Q_{14} Q_{47}}{2\left(E_{(+) 7}-E_{(-) 1}\right)}-\frac{R_{71} R_{14}^{+} R_{47}}{2\left(E_{(+) 4}-E_{(-) 1}\right)} \\
& -\frac{R_{71} R_{14}^{+} R_{47}}{\left(E_{(+) 7}-E_{(-) 1}\right)\left(\left(E_{(+) 4}-E_{(-) 1}\right)\right.}
\end{aligned}
$$

Similar terms evidently arise also within the expression for the dipole $p_{(3) 7}^{\mathrm{o}}$ of the parent hydrocarbon. It is seen that orbitals of three bonds, namely of $\mathrm{Z}_{\mathrm{a}}-\mathrm{C}_{\alpha}, \mathrm{C}_{\alpha}-\mathrm{C}_{\beta}$ and $\mathrm{C}_{\beta}-\mathrm{C}_{\gamma}\left(\mathrm{C}_{\beta}-\mathrm{H}\right)$ bonds, participate in the formation of the third order dipole moment of the $\mathrm{C}_{\beta}-\mathrm{C}_{\gamma}\left(\mathrm{C}_{\beta}-\mathrm{H}\right)$ bond under the accepted approxi- mation. It is also seen that only one of the three resonance parameters contained within each term of Eq. (34), viz. either $Q_{17}$ or $R_{71}$, changes its sign when passing from the cis- to trans-arranged $\mathrm{Z}_{\mathrm{a}}-\mathrm{C}_{\alpha}$ and $\mathrm{C}_{\beta}-\mathrm{C}_{\gamma}\left(\mathrm{C}_{\beta}-\mathrm{H}\right)$ bonds (see Eq. (11)). Hence, the third order induced dipole moment of the $\mathrm{C}_{\beta}-\mathrm{C}_{\gamma}\left(\mathrm{C}_{\beta}-\mathrm{H}\right)$ bond depends on its spatial arrangement with respect to the $\mathrm{Z}_{\mathrm{a}}-\mathrm{C}_{\alpha}$ bond in contrast to second order dipole moment shown in Eq. (9).

Let us introduce the following notations:

$\left(E_{(+) 4}-E_{(-) 1}\right)=\left(E_{(+) 7}-E_{(-) 1}\right)=1+\varepsilon<2$,

$\varepsilon^{\mathrm{o}}=1$

and

$\frac{1}{2(1+\varepsilon)}+\frac{1}{(1+\varepsilon)^{2}}=\sigma>\frac{1}{2}, \quad \sigma^{\mathrm{o}}=\frac{1}{2}$.

Then the respective alteration in the population of the $\mathrm{HAO} \chi_{N+7}$ corresponding to the $\mathrm{C}_{\gamma}(\mathrm{H})$ atom takes the form

$$
\begin{aligned}
-\delta p_{(3) 7}^{(\mathrm{a})} \approx & \sigma\left(S_{74} R_{41} Q_{17}+R_{71} R_{41} R_{47}\right) \\
& -\frac{1}{2}\left(S_{74}^{\mathrm{o}} R_{41}^{\mathrm{o}} Q_{17}^{\mathrm{o}}+R_{71}^{\mathrm{o}} R_{41}^{\mathrm{o}} R_{47}^{\mathrm{o}}\right) \\
& -\frac{1}{2(1+\varepsilon)}\left(R_{74} Q_{41} Q_{17}+R_{71} Q_{14} Q_{47}\right) \\
& +\frac{1}{4}\left(R_{74}^{\mathrm{o}} Q_{41}^{\mathrm{o}} Q_{17}^{\mathrm{o}}+R_{71}^{\mathrm{o}} Q_{14}^{\mathrm{o}} Q_{47}^{\mathrm{o}}\right)
\end{aligned}
$$

(Note that the lower sign of Eq. (1) corresponds to HAO $\left.\chi_{N+7}\right)$.

After taking into account the inequalities of Eqs. (11), (23) and (29) along with the following relations:

$S_{74}=S_{74}^{\mathrm{o}}, R_{41}<R_{41}^{\mathrm{o}}, Q_{17}^{\text {cis }}<Q_{17}^{\mathrm{ocis}}$,

$R_{71}^{\mathrm{cis}}<R_{71}^{\mathrm{ocis}}, R_{47}=R_{47}^{\mathrm{o}}, R_{74}=R_{74}^{\mathrm{o}}$,

$Q_{41}>Q_{41}^{\mathrm{o}}, Q_{47}=Q_{47}^{\mathrm{o}}$

the positive (negative) sign of the term $-\delta p_{(3) 7}^{(\mathrm{a})}$ follows for cis (trans)-arranged $\mathrm{Z}_{\mathrm{a}}-\mathrm{C}_{\alpha}$ and $\mathrm{C}_{\beta}-$ $\mathrm{C}_{\gamma}\left(\mathrm{C}_{\beta}-\mathrm{H}\right)$ bonds, i.e.

$-\delta p_{(3) 7}^{(\mathrm{a}) c i s}>0, \quad-\delta p_{(3) 7}^{(\mathrm{a}) \operatorname{trans}}<0$.

Hence, the third order dipole moment contributes to an additional withdrawal of population from HAO $\chi_{N+7}$ of the trans $-\mathrm{C}_{\beta}-\mathrm{C}_{\gamma}\left(\mathrm{C}_{\beta}-\mathrm{H}\right)$ bond and to an 
additional increase of population of the same $\mathrm{AO}$ in the cis-arranged $\mathrm{C}_{\beta}-\mathrm{C}_{\gamma}\left(\mathrm{C}_{\beta}-\mathrm{H}\right)$ bond. As a result, the trans-effect of the heteroatom $\mathrm{Z}_{\mathrm{a}}$ upon the $\mathrm{C}_{\gamma}$ atoms proves to be additionally strengthened.

In the case of an electron-donating substituent $\left(\mathrm{Z}_{\mathrm{d}}\right)$, the BOs $\varphi_{(+) 1}, \varphi_{(+) 4}$ and $\varphi_{(-) 4}$ should be considered as mediators of the indirect interaction between the BBO $\varphi_{(+) 7}$ and the $\mathrm{ABO} \varphi_{(-) 7}$. As a result, the principal contributions to the third order dipole $p_{(3) 7}^{(\mathrm{d})}$ are

$$
\begin{aligned}
& p_{(3) 7}^{(\mathrm{d})} \sim \frac{S_{71} S_{14} R_{47}}{2\left(E_{(+) 1}-E_{(-) 7}\right)}+\frac{S_{74} S_{41} R_{17}}{2\left(E_{(+) 1}-E_{(-) 7}\right)} \\
& -\frac{S_{71} R_{14} Q_{47}}{\left(E_{(+) 1}-E_{(-) 7}\right)\left(E_{(+) 1}-E_{(-) 4}\right)}-\frac{S_{71} R_{14} Q_{47}}{2\left(E_{(+) 1}-E_{(-) 4}\right)} \\
& -\frac{R_{74} R_{41}^{+} R_{17}}{\left(E_{(+) 1}-E_{(-) 4}\right)\left(E_{(+) 1}-E_{(-) 7}\right)}-\frac{R_{74} R_{41}^{+} R_{17}}{2\left(E_{(+) 1}-E_{(-) 4}\right)} .
\end{aligned}
$$

After introducing the designations

$$
\left(E_{(+) 1}-E_{(-) 7}\right)=\left(E_{(+) 1}-E_{(-) 4}\right)=1+\lambda<2,
$$

$\lambda^{\mathrm{o}}=1$

$$
\frac{1}{2(1+\lambda)}+\frac{1}{(1+\lambda)^{2}}=\kappa>\frac{1}{2}, \kappa^{\mathrm{o}}=\frac{1}{2}
$$

the difference $-\delta p_{(3) 7}^{(\mathrm{d})}$ determining the population alteration of the HAO $\chi_{N+7}$ becomes

$$
\begin{aligned}
-\delta p_{(3) 7}^{(\mathrm{d})}= & \kappa\left(S_{71} R_{14} Q_{47}+R_{74} R_{14} R_{17}\right) \\
& -\frac{1}{2}\left(S_{71}^{\mathrm{o}} R_{14}^{\mathrm{o}} Q_{47}^{\mathrm{o}}+R_{74}^{\mathrm{o}} R_{14}^{\mathrm{o}} R_{17}^{\mathrm{o}}\right) \\
& -\frac{1}{2(1+\lambda)}\left(S_{71} S_{14} R_{47}+S_{74} S_{41} R_{17}\right) \\
& +\frac{1}{4}\left(S_{71}^{\mathrm{o}} S_{14}^{\mathrm{o}} R_{47}^{\mathrm{o}}+S_{74}^{\mathrm{o}} S_{41}^{\mathrm{o}} R_{17}^{\mathrm{o}}\right) .
\end{aligned}
$$

If we take into account the inequalities of Eqs. (11), (23), (29) and (38) along with the relations

$$
S_{71}^{c i s}>S_{71}^{\text {ocis }}, R_{14}<R_{14}^{\mathrm{o}}, R_{17}^{\text {cis }}>R_{17}^{\mathrm{ocis}}, S_{14}>S_{14}^{\mathrm{o}}
$$

contributions of the third order dipole moments to the population of $\mathrm{HAO} \chi_{N+7}$ are negative (positive) for the cis (trans)-arranged $\mathrm{C}_{\beta}-\mathrm{C}_{\gamma}\left(\mathrm{C}_{\beta}-\mathrm{H}\right)$ bonds, i.e.

$$
-\delta p_{(3) 7}^{(\mathrm{d}) i s}<0, \quad-\delta p_{(3) 7}^{(\mathrm{d}) \text { trans }}>0 .
$$

It is seen that the HAO $\chi_{N+7}$ of the trans-arranged $\mathrm{C}_{\beta}-\mathrm{C}_{\gamma}\left(\mathrm{C}_{\beta}-\mathrm{H}\right)$ bond acquires an additional population owing to the third order intrabond polarization, and this leads to an increase of the trans-electron-donating effect of the heteroatom $Z_{d}$ upon the $C_{\gamma}(H)$ atoms.

Therefore, the third order interbond charge transfer and the respective induced dipole moment may be considered as additive components of the trans-effect of heteroatom.

Before finishing this section, let us also note that terms within the first parentheses of the right-hand sides of Eqs. (37) and (42) are likely to be those determining the third order induced dipole moments (this expectation may be supported by Eqs. (36) and (41), respectively). These terms, however, do not coincide with the simple products of three resonance parameters determining the third order charge transfer (Section 3). Hence, no predominant increment containing a simple product of three resonance parameters may be revealed within the trans-effect of heteroatom if both additive components of the effect are taken into consideration.

\section{Comparison to the inductive effect and conclusions}

The results of the present contribution along with those of Refs. [21-24] yield a unified description of the inductive effect of a heteroatom and of its transeffect, viz. these effects are represented by terms of the same power series for occupation numbers. On this basis, the following common features of the effects have been revealed:

1. The additive components of both effects are similar in their nature. Indeed, the trans-effect of heteroatom consists of the interbond charge transfer and of the secondary polarization of the bond under study under the influence of the $\mathrm{Z}-\mathrm{C}_{\alpha}$ bond, as it was the case with the inductive effect $[23,24]$.

2. The above-mentioned additive components and thereby the effects under discussion both may be expressed and interpreted in terms of direct (through-space) and indirect (through-bond) interactions of BOs.

On the other hand, the inductive effect of a 
heteroatom and the relevant trans-effect are related to terms of different order within the power series for occupation numbers, namely, to the second and to the third order terms, respectively. This fact forms the basis for their individual features:

1. The particular interorbital interactions determining the additive components of the trans-effect differ from those of the inductive effect [23,24]. Thus, the increment of the interbond charge transfer to the trans-effect of heteroatom is proportional to product of the direct interaction between orbitals of the $\mathrm{Z}-\mathrm{C}_{\alpha}$ and $\mathrm{C}_{\beta}-\mathrm{C}_{\gamma}\left(\mathrm{C}_{\beta}-\mathrm{H}\right)$ bonds and of their indirect interaction predominantly by means of orbitals of the $\mathrm{C}_{\alpha}-\mathrm{C}_{\beta}$ bond, whereas the relevant increment to the inductive effect depends on square of the direct interaction of the same orbitals [23,24]. The second and the third order induced dipole moment of the $\mathrm{C}-\mathrm{C}(\mathrm{C}-\mathrm{H})$ bonds under the influence of the $\mathrm{Z}-\mathrm{C}_{\alpha}$ bond both are related to the difference in the indirect interaction between the two BOs of the bond under study before and after substitution but the numbers of mediators of these interactions do not coincide with one another (these equal to one and two, respectively).

2. The above-described dissimilarity, in turn, proves to be accompanied by different role of signs of overlap integrals (and thereby of resonance parameters) between $\mathrm{BOs}$ of the $\mathrm{Z}-\mathrm{C}_{\alpha}$ bond and of the bond under study in the formation of the final populations of orbitals of the latter, viz. these signs play no part in the case of the inductive effect but their role becomes a decisive one if we turn to the transeffect. This conclusion may be traced back to the fact that the second order (square) dependence of additive components of the inductive effect upon the above-mentioned resonance parameters becomes replaced by a first order (linear) dependence when passing to the trans-effect.

On the whole, the unified description of the inductive and of the trans-effect of heteroatom by means of the power series for occupation numbers allows the two effects to be considered as particular manifestations of the heteroatom influence. Again, the abovementioned representation of these particular aspects by the second and third order terms of this series indicates certain hierarchy of the effects.

\section{References}

[1] F. Bohlman, Chem. Ber. 90 (1958) 2157.

[2] H.B. Schlegel, S. Wolf, F. Bernardi, J. Chem. Phys. 67 (1977) 4181.

[3] J.B. Lambert, R.G. Keske, J. Am. Chem. Soc. 88 (1966) 620.

[4] M.W. Mackenzie, Spectrochim. Acta, A 40 (1984) 279.

[5] D.B. Shatkovskaya, V.L. Gineityte, A.B. Bolotin, Theoretitcheskaya i Eksp, Khimia 22 (1986) 168.

[6] V. Bachler, G. Olbrich, Theor. Chem. Acta 57 (1980) 329.

[7] I.A. Shlygina, V.Z. Gabdrakipov, Zhurn. Fizitcheskoi Khimii 57 (1983) 2618.

[8] C.K. Ingold, Structure and Mechanism in Organic Chemistry, Cornell University Press, Ithaca, NY, 1953.

[9] E.S. Gould, Mechanism and Structure in Organic Chemistry, Holt, New York, 1959.

[10] A.S. Dneprovskii, T.I. Temnikova, Theoretical Fundamentals of Organic Chemistry, Khimia, Leningrad, 1991.

[11] G. Klopman (Ed.), Chemical Reactivity and Reaction Paths Wiley, New York, 1974.

[12] J. Moshida, J. Take, Y. Saito, Y. Yoneda, J. Org. Chem. 32 (1967) 3894.

[13] V.L. Gineityte, Zhurn. Strukt. Khim. 29 (1988) 164.

[14] V.L. Gineityte, D.B. Shatkovskaya, Lithuanian. J. Phys. 30 (1990) 658.

[15] D. Satkovskiene, V. Gineityte, Int. J. Quant. Chem. 58 (1996) 453.

[16] F. Weinhold, T.K. Brunck, J. Am. Chem. Soc. 98 (1976) 3745.

[17] M.M. Mestetchkin, Method Matricy Plotnosti v Teorii Molekul, Naukova Dumka, Kiev, 1977.

[18] V. Gineityte, D. Shatkovskaya, J. Mol. Struct. (Theochem) 201 (1989) 49.

[19] V. Gineityte, D. Shatkovskaya, Croat. Chem. Acta 62 (1989) 661.

[20] V. Gineityte, D. Shatkovskaya, Int. J. Quant. Chem. 39 (1991) 11.

[21] V. Gineityte, J. Mol. Struct. (Theochem) 343 (1995) 183.

[22] V. Gineityte, J. Mol. Struct. (Theochem) 430 (1998) 97.

[23] V. Gineityte, J. Mol. Struct. (Theochem) 364 (1996) 85.

[24] V. Gineityte, J. Mol. Struct. (Theochem) 434 (1998) 43. 